\title{
TWO-DIMENSIONAL SIMULATION TO INVESTIGATE THE INTERACTION OF FLUID - STRUCTURE INSIDE A MICROCHANNEL
}

\section{WITH ELASTIC AND RIGID BOUNDARY}

\author{
AS'AD ALIZADEH ${ }^{1 *} \&$ ANAS ABID MATTIE ${ }^{2}$ \\ ${ }^{I}$ Department of Mechanical Engineering, College of Engineering, University of Zakho, Zakho City, Iraq \\ ${ }^{2}$ Department of Mechanics, Duhok Polytechnic University, Duhok, Kurdistan Region, Iraq
}

\begin{abstract}
In this paper, shape change of the elastic and rigid boundary in a micro-channel is simulated. The rings are considered as elastic boundaries immersed in the fluid flow. In this study, we use the Immersed Interface Method to simulate the flow around rigid objects and movement of the flexible objects. In this method, there is no compulsion to match the object mesh and the flow mesh. The results were found to be in good agreement with available data. First, the simultaneous motion of multiple membranes in a micro-channel and their interaction with each other and with flow are evaluated. In addition, simultaneous presence of multiple membranes would result in a reduction in the flow velocity. Finally, the effects of changes in the elastics and bending moduli on the deformation of cell are considered.

KEYWORDS: Elastic Ring, Immersed Interface Method, Interaction \& Deformation
\end{abstract}

Received: Apr 29, 2018; Accepted: May 19, 2019; Published: Jul 19, 2019; Paper Id.: IJMPERDAUG2019119

\section{INTRODUCTION}

The immersed interface method (IIM) is the most appropriate approach for solving the problem of fluid-solid interaction. The basis of IIM is to add a forcing term as a source term to the Navier-Stokes [1]. Feng and Michaelides [2] were the first to IIM and simulated suspensions of rigid disks in 2D. Le and Zhang [3] used in their work a hybrid LBM- IIM and noticed that the computed velocity profiles can deviate greatly from theoretical ones even for very simple flow situations, both in the immersed boundary layer and the bulk region. Dupuis et al. [4] studied how the coupling method of the forcing term between the Eulerian and Lagrangian grids could affect the results for the flow over an impulsively started cylinder at moderate Reynolds (Re) number. Wu and Shu [5] proposed a new version of IIM, which could well consider the effect of external force on the momentum flux as well as the discrete lattice effect. JiSeok and SangHwan [6] presented a numerical scheme for fluid-structure interaction, especially for elastic structures. They employed a hybrid IIM using an improved direct forcing scheme for the fluid, and a finite element method with Euler beam elements for the elastic plate. Zhang et al. [7, 8] also used a combination of the IIM and the LBM to investigate the microscopic hemodynamic and hemorheological behaviors of discrete RBCs in shear flow. They noted that three-dimensional simulation of RBCs is required to attain accurate results. In the present work, first, the simultaneous motion of multiple membranes in a microchannel and their interaction with each other and with flow are evaluated. Finally, the effects of changes in the elastics and bending moduli on the deformation of cell are considered. 


\section{GOVERNING EQUATIONS}

Navier-Stokes equations for the incompressible flow are as follows:

$$
\begin{aligned}
& \nabla \cdot \vec{u}=0 \\
& \rho\left(\frac{\partial \vec{x}}{\partial t}+\vec{u} \cdot \nabla \vec{u}\right)=-\nabla p+\eta \nabla^{2} \vec{u}+\vec{F}
\end{aligned}
$$

With boundary and initial condition [9]:

$$
\begin{aligned}
& u \|_{a E}=u_{b} \\
& u(x, 0)=u_{b}
\end{aligned}
$$

Where, $u$ is the fluid velocity, $p$ is the pressure, $\rho$ is the density, and $\mu$ the viscosity of the fluid. The force $F$ which has the form

$$
\vec{F}(\vec{x}, t)=\int_{r(s)} f(s, t) \delta(\vec{x}-\vec{X}(s, t)) d s
$$

The motion of the interfaces is shown as below formulation

$$
\vec{u}(\vec{X}(s, t), t)=\frac{a \vec{A}(s, t)}{\partial t}=\int_{E} \vec{u}(\vec{x}, t) \delta(\vec{x}-\vec{A}(s, t)) d \vec{x}
$$

The force density using an expression of the form,

$$
\vec{f}(\mathrm{~g}, \mathrm{t})=\mathrm{k}\left(\vec{X}^{*}(\mathrm{~g})-\vec{X}(\mathrm{~g}, \mathrm{t})\right)
$$

The force is expressed as

$$
\vec{f}(s, t)=\alpha \int_{0}^{t} \vec{v}\left(s, t^{r}\right) d t^{r}+\beta \vec{v}(s, t)
$$

The respective normal and tangential components of the force density $f_{1}=\vec{f}(s, t), \bar{n}$ and $f=\vec{f}(s, t), \vec{t}$ can be related to the jump conditions for pressure and velocity as follows [8]:

$$
\begin{aligned}
& {[\vec{u}]=0,\left[\mu \vec{u}_{\xi}\right]=-f_{2} \bar{z},\left[\vec{u}_{\tau}\right]=0} \\
& {[p]=f_{1},\left[p_{\xi}\right]=\frac{\partial f_{1}}{\partial s},\left[p_{\eta}\right]=\frac{a f_{1}}{g_{s}}}
\end{aligned}
$$

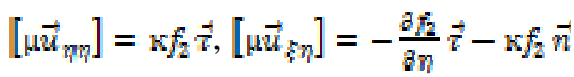

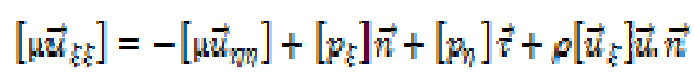

We consider the below relations as a simple

$$
\begin{aligned}
& {\left[\vec{u}_{x}\right]=\left[\vec{u}_{\xi}\right] n_{1}+\left[\vec{u}_{\eta}\right] \tau_{1}} \\
& {\left[\vec{u}_{y y}\right]=\left[\vec{u}_{\xi \xi}\right] n_{2}^{2}+2\left[\vec{u}_{\xi \eta}\right] n_{2} \tau_{2}+\left[\vec{u}_{\xi \eta}\right] \tau_{\frac{2}{2}}^{2}}
\end{aligned}
$$




\section{RESULTS AND DISCUSSIONS}

\section{Validation}

In this section, we investigate the interaction of the flow and a circular ridge cylinder located at the center of the channel. Vortex shedding frequency is $f_{s}$. The Strouhal number $\left(\mathrm{St}=\frac{D f_{s}}{u_{\infty}}\right)$ and the average drag coefficient are compared with other results in Table 1, which is in good agreement. It can be seen that with the increase in Reynolds number, the average drag coefficient decreases, but the Strouhal number increases. Increasing the Reynolds number increases the rate of the vortices spreading. That is, in a certain time, more vortices are spread in the flow.

Table 1 Comparison of the present results with other results

\begin{tabular}{|l|l|c|c|}
\cline { 3 - 4 } \multicolumn{2}{c|}{} & $\overline{\mathrm{C}}_{\mathfrak{D}}$ & $\mathrm{St}$ \\
\hline \multirow{4}{*}{$\mathrm{Re}=100$} & Braza et al.[10] & 1.359 & 0.16 \\
\cline { 2 - 4 } & Ji et al.[11] & 1.376 & 0.169 \\
\cline { 2 - 4 } & present result & 1.380 & 0.16 \\
\hline \multirow{3}{*}{$\operatorname{Re}=200$} & Braza et al.[10] & 1.386 & 0.20 \\
\cline { 2 - 4 } & Ji et al.[11] & 1.354 & 0.20 \\
\cline { 2 - 4 } & present result & 1.355 & 0.20 \\
\hline
\end{tabular}

\section{Simultaneous Motion of Three Membranes}

In this section, effect of membrane's location on each other is investigated, Three membranes were placed on the micro- channel's axis of symmetry (Figure 1a) in Figure 1b, deformation of the membrane at time $t^{*}=0.5$ is observed. It is observed that pressure and velocity at top and bottom of membrane's center had symmetry. So, there are no pressure differences in the vertical direction so as to deviate membrane from the center of the micro-channel. The low pressure difference created around the membranes at x-direction would create a force from fluid to membrane in fluid motion direction from left to right. As can be seen, all three membranes would gain more convexity in front and more concavity from behind, so as to overcome the flow resistance. This result can also be seen in experimental results of $[12,13]$.

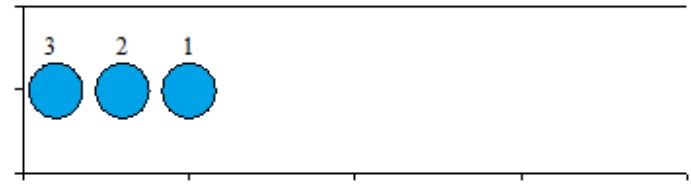

(a)

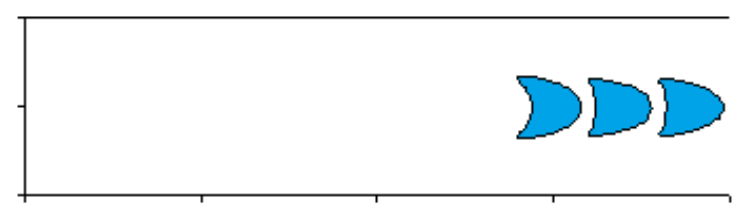

(b)

Figure 1: (a) Initial Position of the Three Membranes, (b) Their Motion and Deformation at Time $T^{*}=0.5$ 


\section{The Effect of Increasing the Reynolds Number on the Flow Pattern}

The problem geometry in this case is shown in Figure 2. All dimensions of the cylinders are dimensionless by the length of the square obstacle. The length of the flow input (the distance between the front edge of the square cylinder and the channel entrance) is L / 5. The Reynolds number is Re <300. Figure 2 shows the flow lines for several different Reynolds numbers. When $\mathrm{Re}=1$, due to the predominance of Viscose forces, a steady flow will be formed without any separation. As the Reynolds number increases, separation of the flow occurs in the back of the barrier. The onset of the separation phenomenon and the formation of stationary vortices for the pre-sent problem is $\operatorname{Re}=3$ (Figure 2a). As can be seen, this value is for a square barrier smaller than the corresponding value for the flow around the circular cylinder. The reason for the smaller value of this value in the flow around the square barrier can be related to the existence of sharp edges in this problem. When the Reynolds number of the upstream flow reaches the critical Reynolds number, alternating unsteady vortex phenomenon occurs. The critical Reynolds number for the studied issue is calculated with blockage ratio of 0.125 , about 55. References [14, 15] have provided 54 and 70 respectively for critical values of Reynolds. Figure $4 \mathrm{~b}$ shows the stream lines for $\operatorname{Re}=200$. Since the flow in this case is unsteady, the shape of the stream lines is drawn in a special moment. Flow in Re $=200 \mathrm{In}$ addition to the vor-tex flow behind the cylinder, smaller vortex flows will be formed adjacent to the lateral edges of the cylinder. In other words, the separation region from the back of the cylinder penetrates to the front edge. Based on the results of the calculations, the penetration phenomenon of the separation region to the front edge can be seen in the Reynolds range greater than 130. This phenomenon has significant effects on the flow characteristics that will be addressed in the following sections.

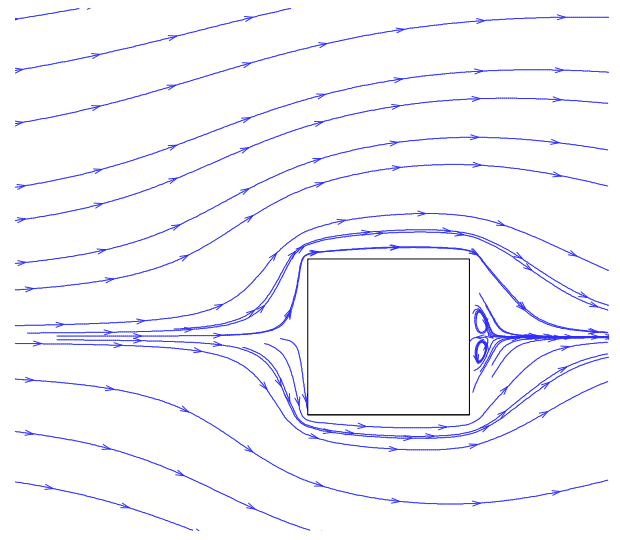

(a)

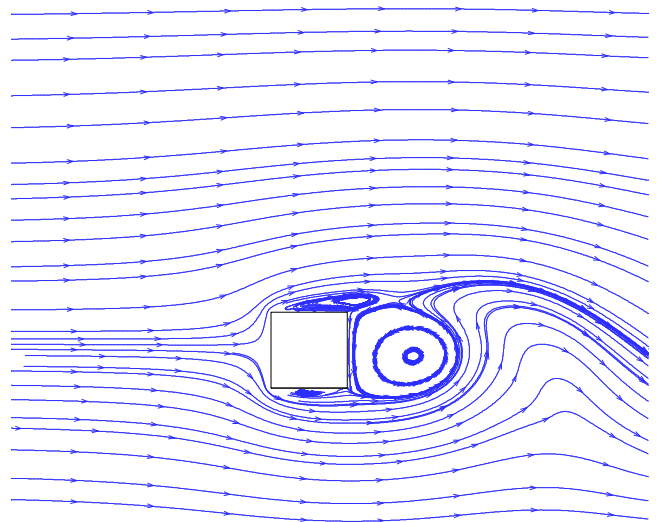

(b)

Figure 2: The Stream Lines for a) $\operatorname{Re}=3, \mathrm{~b}) \mathrm{Re}=200$

Figure 3 shows the variation in vortex region length (steady flow with symmetric vortices) versus Reynolds number. The calculations confirm the linear increase in vortex region length in terms of Reynolds number increase. 


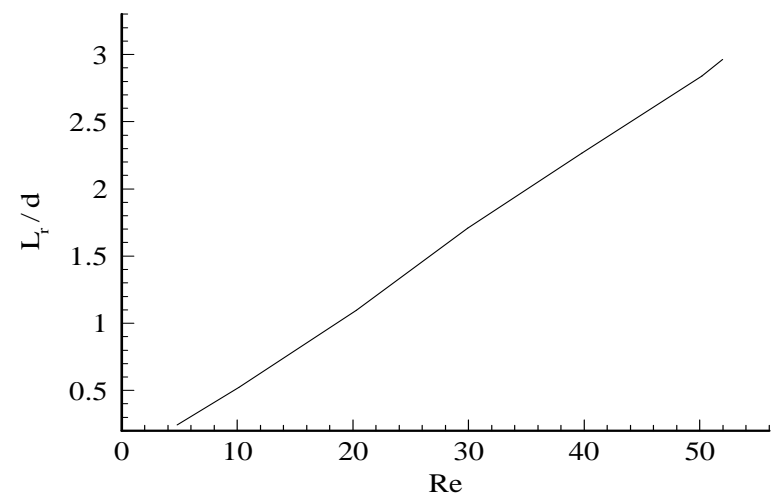

Figure 3: The Vortex Region Length Versus the Reynolds Number in the Permanent Flow

\section{CONCLUSIONS}

In this study, we use the Immersed Interface Method to simulate the flow around rigid objects and movement of the flexible objects. In this method, there is no compulsion to match the object mesh and the flow mesh. The results were found to be in good agreement with available data. First, the simultaneous motion of multiple membranes in a micro-channel and their interaction with each other and with flow are evaluated. The numerical simulation of the flow of fluid through one or a set of objects that causes the flow to separate from the surface of them has been the subject of interest by researchers over the past few decades. One of the most important types of these objects is those with a square cross section, which have important and diverse applications in different industries. The flow around a square cylinder was simulated and we surveyed different flow patterns. The changes in the vortex region length and the Drag coefficient were investigated in different Reynolds.

\section{REFERENCES}

1. H. K. Versteeg and W. Malalasekera, "An Introduction to Computational Fluid Dynamics", Second Edition, 2007, London.

2. Y. T. Feng, K. Han, D. R. J. Owen, Coupled lattice Boltzmann method and discrete element modeling of ring transport in turbulent fluid flows, Int.J.Numer.Methods Eng.72 (2007)193-206.

3. J. F. Zhang, G. G. Le, Boundary slip from the immersed boundary lattice Boltzmann models, Phys. Rev.E. 79( 2009)046701.

4. A. Dupuis, P. Chatelain, P. Koumoutsakos, An immersed boundary-lattice-Boltzmann method for the simulation of the flow past an impulsively started cylinder, J.Comput.Phys.227(2008) 4486-4498.

5. J. Wu, C. Shu, Implicit velocity correction-based immersed boundary-lattice Boltzmann method and its applications, J. Comput. Phys. 228(2009) 1963-1979.

6. L.Jiseok, S. Lee, Flow Around A Elastic Plate In A Free Stream, J.Mech. Sci.Technology.5 (2011) 379-390

7. J.F. Zhang, P.C. Johnson, A.S. Popel, An immersed boundary lattice Boltzmann approach to simulate deformable liquid capsules and its application to microscopic blood flows, Phys. Biol. 4(2007) 285-295.

8. J.F. Zhang, P.C. Johnson, A.S. Popel, Red blood cell aggregation and dissociation in shear flows simulated bylatticeBoltzmannmethod, J.Biomech.41 (2008)47-55

9. D.L. Brown, R. Cortez, M.L. Minion, Accurate projection methods for the incompressible Navier-Stokes equations, J. Comput. Phys. 168 (2001) 464-499. 
10. M. Braza, P. Chassaing, and H. Ha Minh. Nu-merical study and physical analysis of the pres-sure and velocity fields in the near wake of a circular cylinder.J. Fluid Mech., 165:79-130, 1986.

11. C. Ji, A. Munjiza, and J. J. R. Williams. A novel iterative direct-forcing immersed boundary method and its finite volume applications. J. Comput. Phys.,231(4):1797-1821, 2012

12. Pislaru, C. (2013). A Review on Real-Time Simulation of CNC Machine Tool Dynamics. International Journal of Mechanical Engineering, 2(5), 39-44.

13. R. Skalak, P. I. Branemark, Deformation of red blood cells in capillaries, Science, Vol. 164, No. 11, pp. 163- 196,1969.

14. V. Vitkova, M. Mader, T. Podgorski, Deformation of vesicles flowing through capillaries, Europhysics Letters, Vol. 68, No. 3, pp. 398-405, 2004.

15. Breuer, M., Bernsdorf, J., Zeiser, T., and Durst, F. "Accurate Computations of Laminar Flow Past a Square Cylinder", J. Heat and Fluid Flow, Vol. 2, No. 2, pp.186-196, 2000.

16. Okajima, A. "Strouhal Numbers of Rectangular Cylinders", J. Fluid Mech., Vol. 123, pp. 379-398, 1982. 